
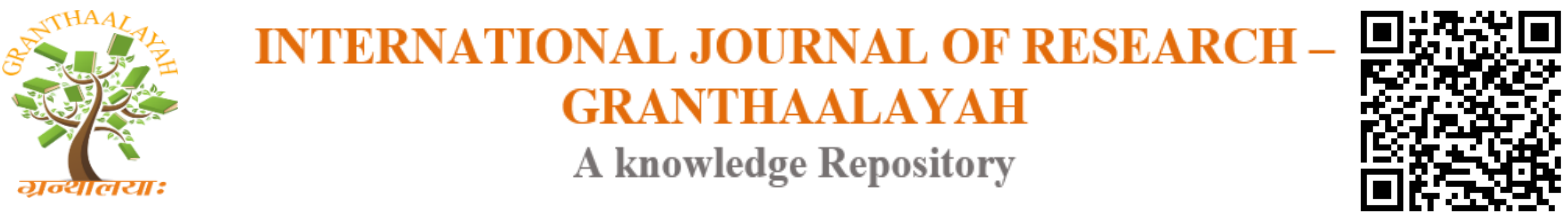

Science

\title{
COMPARISON OF AERODYNAMICS CHARACTERISTICS OF NACA 0015 \& NACA 4415 AEROFOIL BLADE
}

\author{
Rubel, R. I. ${ }^{* 1}$, Uddin, M. K. ${ }^{2}$, Islam, M. Z. ${ }^{3}$, Rokunuzzaman, M. ${ }^{4}$ \\ ${ }^{* 1}$ Department of Mechanical Engineering, Bangladesh Army University of Science and \\ Technology, Saidpur Cantonment-5311, Bangladesh \\ 2, 3,4 Department of Mechanical Engineering, Rajshahi University of Engineering \& Technology, \\ Rajshahi-6204, Bangladesh
}

\begin{abstract}
NACA 0015 and NACA 4415 aerofoil are most common four digits and broadly used aerodynamic shape. Both of the shapes are extensively used for various kind of applications including turbine blade, aircraft wing and so on. NACA 0015 is symmetrical and NACA 4415 is unsymmetrical in shape. Consequently, they have big one-of-a-kind in aerodynamic traits at the side of widespread differences of their utility and performance. Both of them undergo the same fluid principle while applied in any fluid medium giving dissimilar outcomes in aerodynamics behavior. On this work, experimental and numerical investigation of each NACA 0015 and NACA 4415 is done to decide their performance. For this purpose, aerofoil section is tested for a prevalence range attack of angle (AOA). The study addresses the performance of NACA 0015 and NACA 4415 and evaluates the dynamics of flow separation, lift, drag, pressure and velocity contour and so on.
\end{abstract}

Keywords: Aerofoil; CFD; Lift and Drag Force; Pressure; Velocity Contour.

Cite This Article: Rubel R. I., Uddin M. K., Islam M. Z., and Rokunuzzaman M.. (2017). "COMPARISON OF AERODYNAMICS CHARACTERISTICS OF NACA 0015 \& NACA 4415 AEROFOIL BLADE." International Journal of Research - Granthaalayah, 5(11), 187-197. https://doi.org/10.29121/granthaalayah.v5.i11.2017.2346.

\section{Introduction}

Aerofoil is such a streamlined shape or profile that when it travels through air, the air is fallen to pieces in two sections and goes above and beneath the wing. The wing's upper surface is framed so the air hurrying over the top surface velocities up and extends [1]. The stream is quickened over the aerofoil because of the preoccupation of stream from the lower side as appeared in figure 1 [2]. Higher mean speed is seen close to the suction crest area [3, 4]. At the point when an airfoil is moved quickly through an AOA range that incorporates the static stall angle, the angle of maximum lift can be incredibly expanded [5]. The NACA aerofoils are created and develop by National Advisory Committee for Aerodynamics (NACA). 


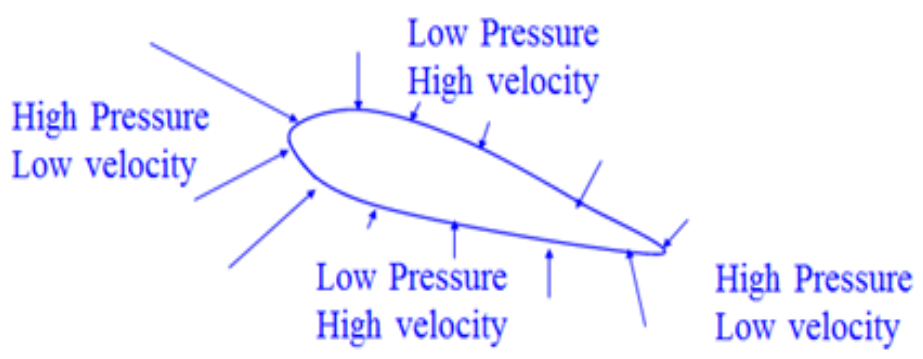

Figure 1: Pressure and Velocity around an aerofoil

NACA 0015 and NACA 4415 profile are appeared underneath and the directions are additionally arranged at the record from which the accompanying profiles are drawn. The NACA 0015 aerofoil is symmetrical with no camber. The digit 15 demonstrates that the aerofoil has a $15 \%$ thickness to chord length proportion; it is $15 \%$ as thick as it is long. The NACA aerofoil 4415 has a greatest camber of $4 \%$ located $40 \%$ ( 0.4 chord) from the main edge with a most maximum thickness of $15 \%$ of the chord. Both NACA 0015 and NACA 4415 aerofoil is examined to comprehend the transient progression of flow separation, lift, drag, pressure and velocity contour. There have been a numerous scientists fascinating in the examination, alteration, and examination of aerofoil. Recently Micro Aerial Vehicles (MAVs) and Unmanned Aerial Vehicles (UAVs) have resuscitated examination enthusiasm on the execution of aerofoil [6]. A symmetrical wing aerofoil is bended on the top to the same degree as it is on the base side. An upper and lower part of the aerofoil is symmetrical when a line is drawn from the focal point of the main edge to the focal point of the trailing edge. This kind of aerofoil is utilized as a part of numerous applications including submarine balances, rotating and some settled wings, air ship vertical stabilizers and so on.

The baseline aerofoil is likewise expected to have NACA 0015 profile [7]. Wind turbine cutting edge additionally utilizes symmetrical NACA 0015 aerofoil [8]. The aerofoil NACA 4415 of optimal design is chiefly utilized as wind turbine blade [9]. Pedro J. Boschetti et. al chips away at the streamlined features advancement and allude the utilization of NACA 0015 with NACA 4415 in a consolidated segment [10]. Near examination of aerofoil NACA 2313 and NACA 7322 utilizing computational fluid flow technique is refined by Umapathi and Soni [11]. A. Spentzos et. al chips away at the 2D and 3D dynamic stall of NACA 0012 and NACA 0015 by CFD (Computational Fluid Dynamics) [12]. NACA 4415 airfoil has a declared impact in decreasing the level of degree of flow separation which is shown by David and Jamey amid working with the oscillation of the upper surface of an aerofoil [13]. Gerontakos and Lee concentrated on the influence of both upward and descending trailing-flap deflections on the speed and vortices flow fields around a NACA 0015 aerofoil subjected to profound deep stall oscillation [14]. Morshed works to find the correlation of the experimental and numerical (CFD) investigation of a Savonius wind turbine [15]. Surface finish condition affect the lift and drag of the aerofoil as the shape and should be considered in aerofoil choice and the expectation of wing attributes [16]. The lift and drag coefficients $\left(C_{L}\right.$ and $\left.C_{D}\right)$ are determine by numerically integrating the pressure distribution around the aerofoil thus friction effect is underestimated and lead to error [17,18]. For the design of aerofoil layout excess wetted area ought to be averted as friction drag is specifically corresponding to the aggregate wetted territory [19]. The mean streamlined chord (MAC) of an aerofoil is utilized to standardize the streamlined strengths to get the lift, drag. 
The 3D stream flow velocity, pressure are measured by joined utilization of subsonic wind tunnel and CFD (FLUENT). An ideal tradeoff between streamlined execution and auxiliary solidness requires a multi-scale enhancement. The general qualities of the both aerofoil geometries are thought about on the premise of AOA yields. The goal in this work focuses on presenting a description of the physics of flow in response to the enhancement of lift and elimination of the drag. The investigation of the huge number of pressure and speed distributions acquired inside the route of the research facilitated for considering the characteristics distinction of flows among the selected aerofoil shapes.

\section{Theoretical and Mathematical Background}

Consider the aerofoil shown in Figure 2. The essential feature of an aerofoil is mean camber line. It is a half way path between upper and lower surface. The extreme forward point is called leading edge and rear point is called trailing edge. Chord line connects the leading and trailing edge point. The difference between the mean camber line and chord line is called camber. Fundamental aerodynamic theory has found adequate for analyzing the essential character of subsonic flow over NACA aerofoil series. The researcher has amassed theoretically and experimentally vast amount of engineering formulation. From physical and mathematical considerations, the incompressible Navier-Stokes equations for Newtonian fluids are presented by [20].

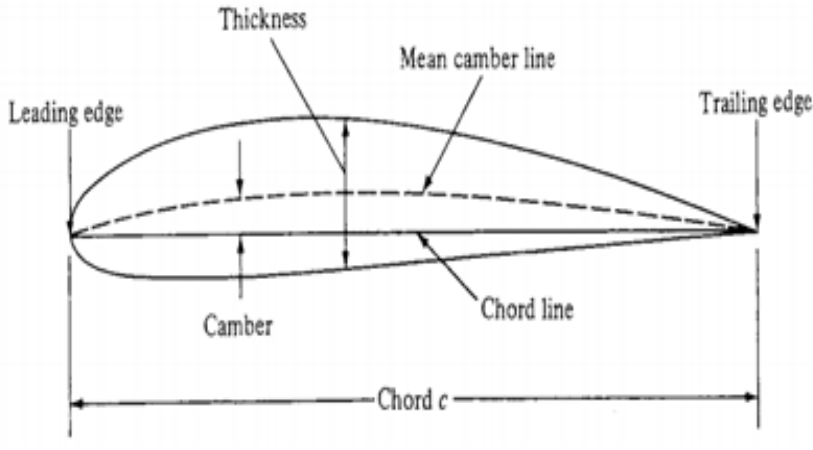

(a)



(b)

Figure 2: (a) Aerofoil; (b) Forces on aerofoil.

$\partial_{\mathrm{t}} \mathrm{v}+(\mathrm{v} \cdot \mathrm{V}) \mathrm{v}-\nabla \cdot(2 \mathrm{vDv})+\nabla_{\mathrm{P}}=\mathrm{f}$

$\nabla \cdot \mathrm{v}=0$

Where $\mathrm{v}$ is the velocity of the flow, $\mathrm{Dv}=(1 / 2)\left(\left(\nabla v+\nabla \mathrm{v}^{\mathrm{t}}\right)\right.$ its deformation tensor, and $\mathrm{p}$ its pressure.

The momentum equation (1) inherited from Newton's law, while equation (2) is the mass conservation equation for incompressible flows. The mass density of fluid $(\rho)$ and velocity $v$ is defined in equation (2). The general mass conservation equation satisfied by $\rho$ and $v$ is

$\delta_{\mathrm{t}} \rho+\nabla \cdot \nabla \mathrm{p}=\mathrm{f}$ 
Moreover, the continuity equation is given as

$$
\frac{\partial \rho}{\partial \mathrm{t}}+\frac{\partial \rho \mathrm{u}}{\partial \mathrm{x}}+\frac{\partial \rho \mathrm{v}}{\partial \mathrm{y}}+\frac{\partial \rho \mathrm{w}}{\partial \mathrm{z}}=0
$$

The mass fluxes in the $\mathrm{x}, \mathrm{y}, \mathrm{z}$ directions are $\rho \mathrm{u}, \rho \mathrm{v}$, and $\rho \mathrm{w}$ respectively. The momentum and the energy equation can be found following the same procedure. The fluxes considered are (a) mass flux $=\rho \mathrm{V}$, (b) flux of $\mathrm{x}, \mathrm{y}$, and $\mathrm{z}$ component of momentum are $\rho \mathrm{uV}, \rho v \mathrm{~V}, \rho w \mathrm{~V}$, (c) flux of

internal energy $=\rho e V$, (d) flux of total energy $=\rho\left(e+\frac{V^{2}}{2}\right)$. For details on these equations see references [21].

When the pressure distribution on the aerofoil surface is known, determining the total lift requires adding up the contributions to the pressure force from local elements of the surface, each with its own local value of pressure [22]. The total lift is therefore the integral of the pressure; within the course perpendicular to some distance field go with the flow, over the entire surface of the aerofoil or wing given by-

$\mathrm{L}=\oint \mathrm{pn} \cdot \mathrm{kdA}$

Where, $\mathrm{L}$ is the lift, $\mathrm{A}$ is the wing surface area, $\mathrm{p}$ is the value of the pressure, $\mathrm{n}$ is the normal unit vector pointing into the wing and $\mathrm{k}$ is the vertical unit vector, normal to the free stream direction. The lift and drag is calculated by the equation given below [23]. Where, the lift coefficient $\left(\mathrm{C}_{\mathrm{L}}\right)$ and the drag coefficient $\left(\mathrm{C}_{\mathrm{D}}\right)$ depend on the angle of attack $\alpha$ (degree).

$\mathrm{C}_{\mathrm{L}}=2 \mathrm{~F}_{\mathrm{L}} /\left(\rho \mathrm{V}^{2} \mathrm{~A}\right)$

$\mathrm{C}_{\mathrm{D}}=2 \mathrm{~F}_{\mathrm{D}} /\left(\rho \mathrm{V}^{2} \mathrm{~A}\right)$

\section{Boundary Conditions and Physical Setup}

The condition that the aerofoil surface is a streamline means that the total velocity component normal to the surface is zero. For laminar $\left(\operatorname{Re}<5 \times 10^{5}\right)$, steady and incompressible flow at velocity $10 \mathrm{~m} / \mathrm{s}$ in a viscous model of air having density around the airfoil has been simulated by solving the equations for conservation of mass and momentum having density $1.225 \mathrm{~kg} / \mathrm{m}$ and viscosity $1.789 \times 10^{5} \mathrm{~kg} / \mathrm{m}$-s at room temperature $288.16 \mathrm{k}$. In a density based steady solution environment convert the governing equations of flow in to algebraic equations that can be solved numerically and the inlet of the system velocity is defined entering at $0^{\circ} \mathrm{AOA}$ (as per the problem statement), at a total magnitude of one. We will also define the gauge pressure at the inlet to be zero. As for the outlet, the only thing can be assumed is that the gauge pressure is zero (Ambient atmospheric condition is imposed at outlet). As for the aerofoil itself, we will treat it like a wall. No slip boundary conditions are imposed. When the pre-calculations are ready, FLUENT can be run to complete the simulation by loading the Fluid Flow (FLUENT) box by dragging and dropping it into the project schematic. Now it is ready to create the geometry for the simulation. Both pressure and velocity measurements are made for angles of attack of $0^{\circ}<$ $\mathrm{AOA}<18^{\circ}$ for NACA 0015 and NACA 4415 at fixed Reynolds number of laminar flow. 


\section{Geometry}

For CFD simulation the coordinates for NACA 0015 and NACA 4415 of the airfoil is imported and the geometry is created that will use for the simulation as in Figure 3(a) and 3(b). In order to analyze fluid flows, flow domains are split into smaller subdomains [24]. Subdomains are made up of geometric primitives like hexahedra and tetrahedral in 3D and quadrilaterals and triangles in 2D [25]. Mesh analysis is performed by assuming relevance center is fine and smoothing is high in C-mesh domain presented in Figure 3(c) and 3(d). External sharp corners, such as those found on the trailing edge of an airfoil, present a challenge in meshing. The governing equations are then discretized to solve the inside each of these subdomains.

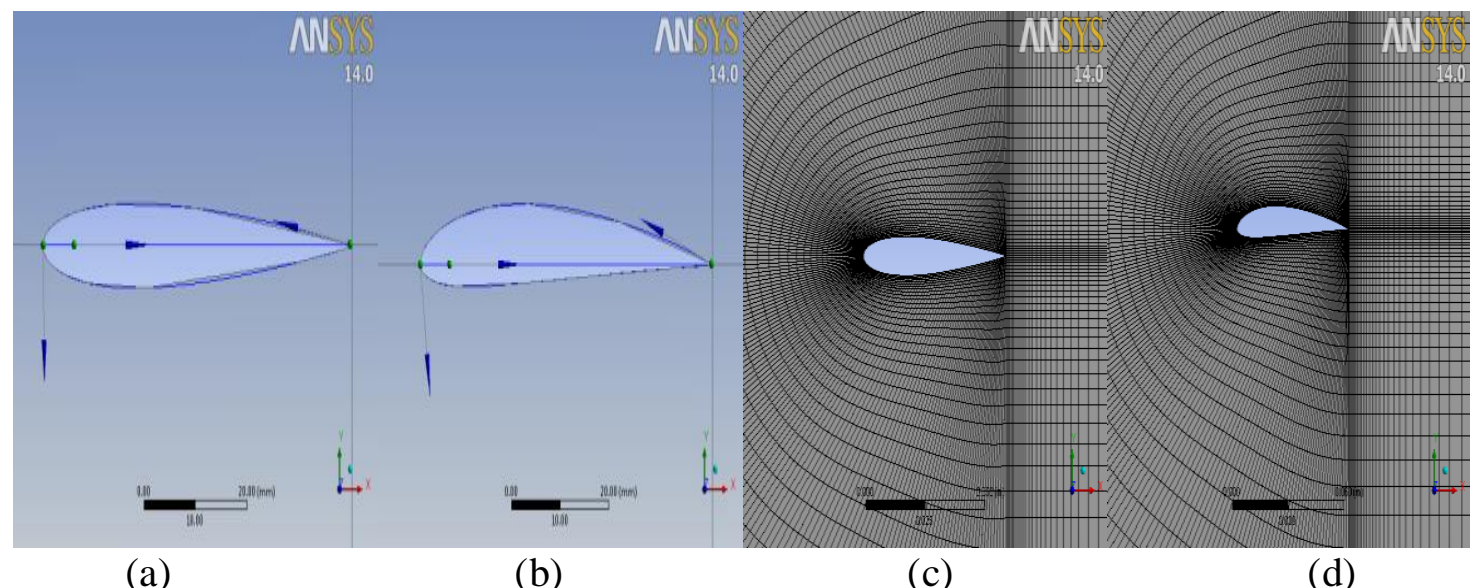

Figure 3: (a) Geometry of NACA 0015; (b) Geometry NACA 4415; (c) Mesh generation for NACA 0015; (d) Mesh generation for NACA 4415.

\section{Results and Discussions}

\subsection{Contours of Static Pressure}

For structural design, estimation of the critical Mach number, moment coefficient knowledge of the pressure distribution over an aerofoil is desirable especially when tests are not available. At zero lift the pressure distributions over the upper and lower surfaces are identical. Contours of static pressure show that static pressure increases at the lower surface of the aerofoil with increasing angle of attack. Figures 4 show the simulation consequences of static pressure for $0^{\circ}$ to $18^{\circ} \mathrm{AOA}$ for viscous model of air as fluid medium. According to the figure underneath at $0^{\circ}$ AOA, NACA 0015 has static pressure of $5.67 \times 10^{1}>5.63 \times 10^{1}$ Pa that obtained for NACA 4415. That clearly indicated that NACA 0015 will have greater pressure gradient at small AOA, so for the lift. The pressure on the lower side of the aerofoil is larger than that of the inward flow stream and effectively pushed the aerofoil upward. Static pressure increases maximum $6.14 \times$ $10^{1} \mathrm{~Pa}$ with $12^{\circ} \mathrm{AOA}$ with quiet laminar flow pattern. 

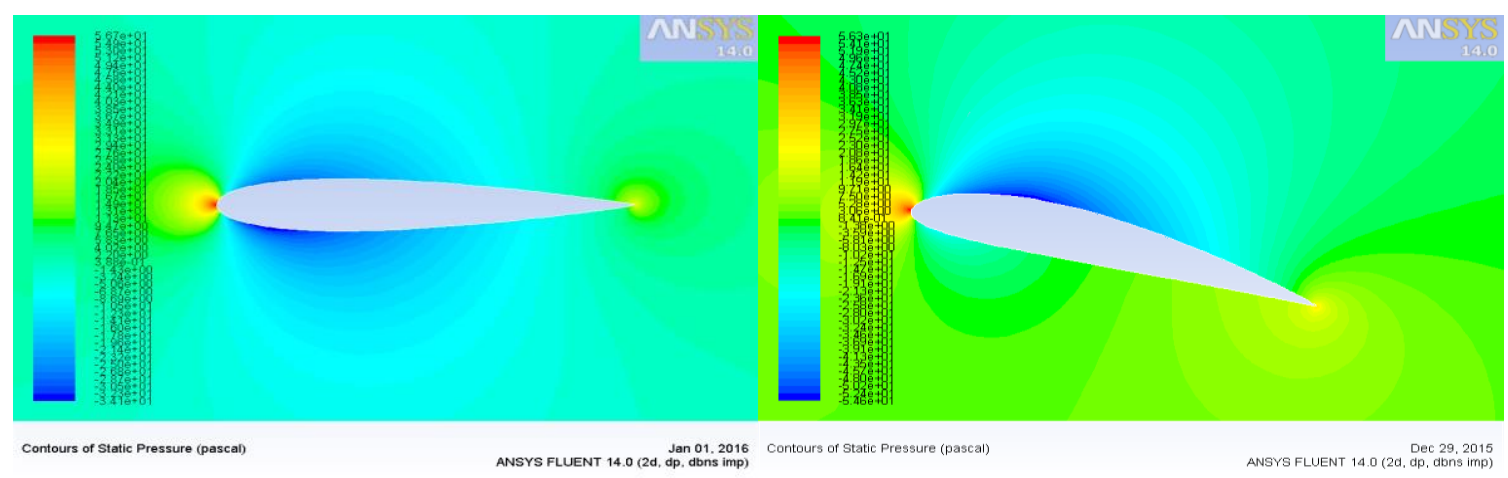

(a) $0^{\circ}$ angle of attack

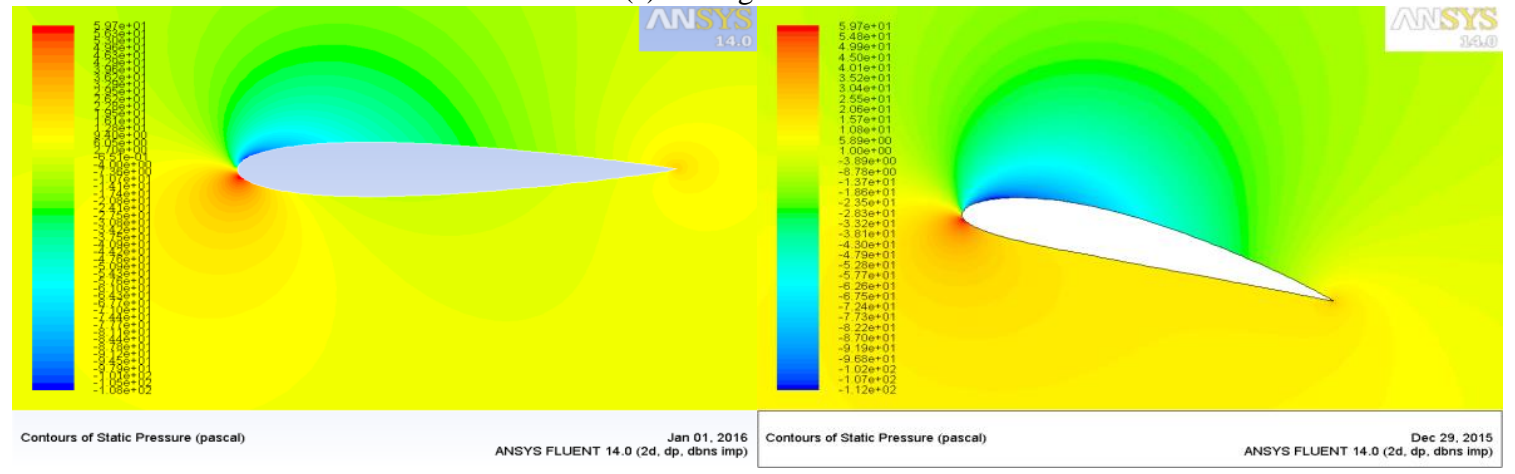

(b) $6^{\circ}$ angle of attack

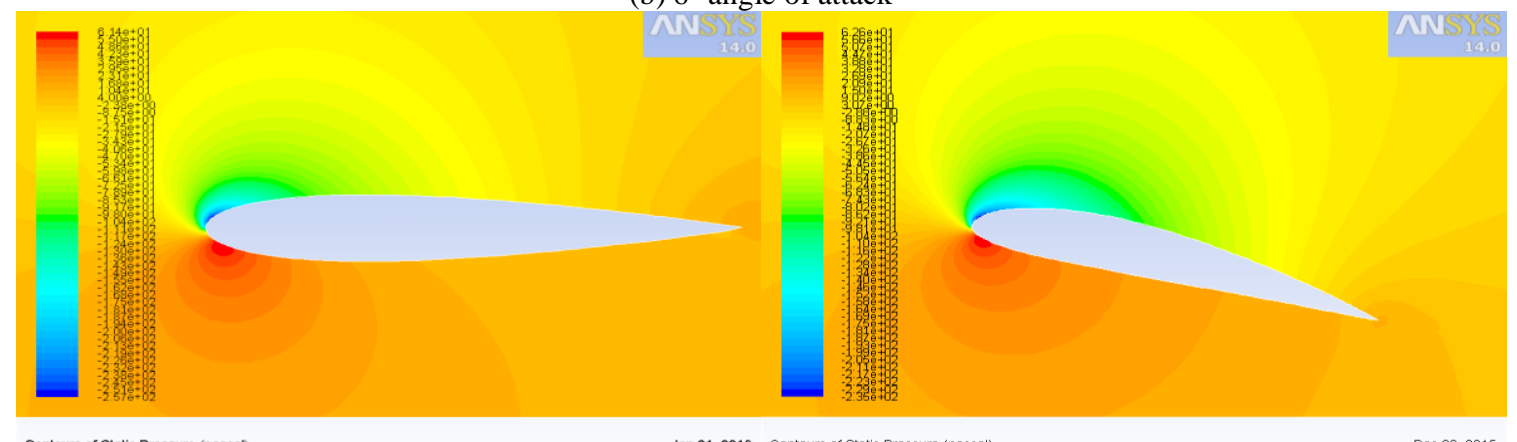

Contours of Static Pressure (pascal)

ANSYS FLUENT 14.0 (2d, Jan, dbns imp 010

ANGYS FLUENT 14.0 (20, Dec 29. 2010nis imp)

(c) $12^{\circ}$ angle of attack

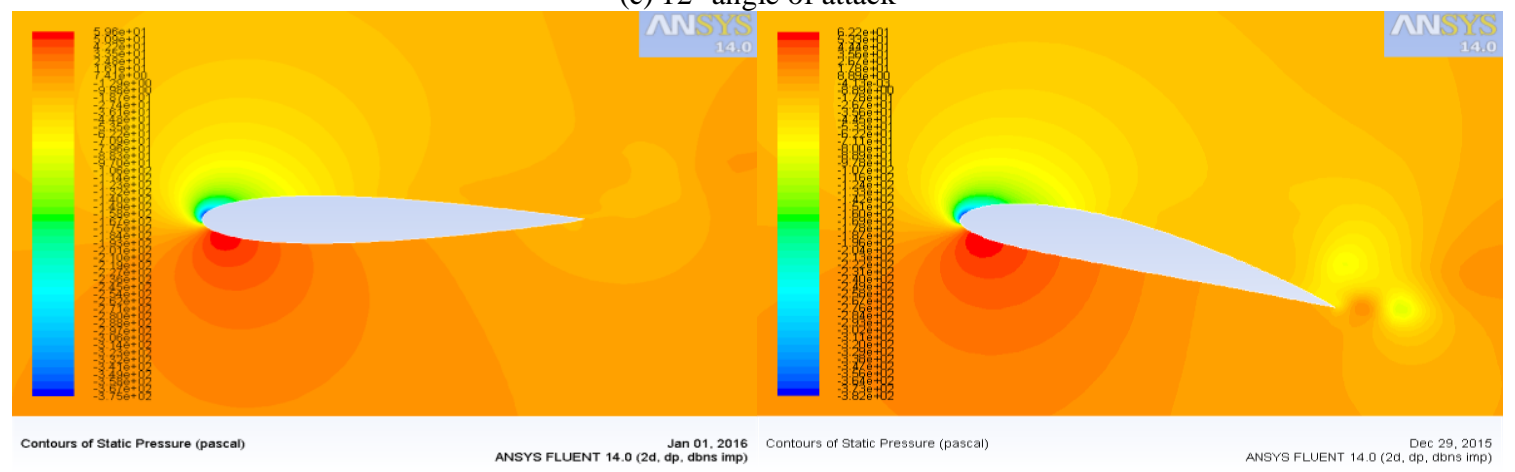

(d) $18^{\circ}$ angle of attack

Figure 4: Comparison pairs of Static Pressure contours for NACA 0015 and NACA 4415 aerofoil. 


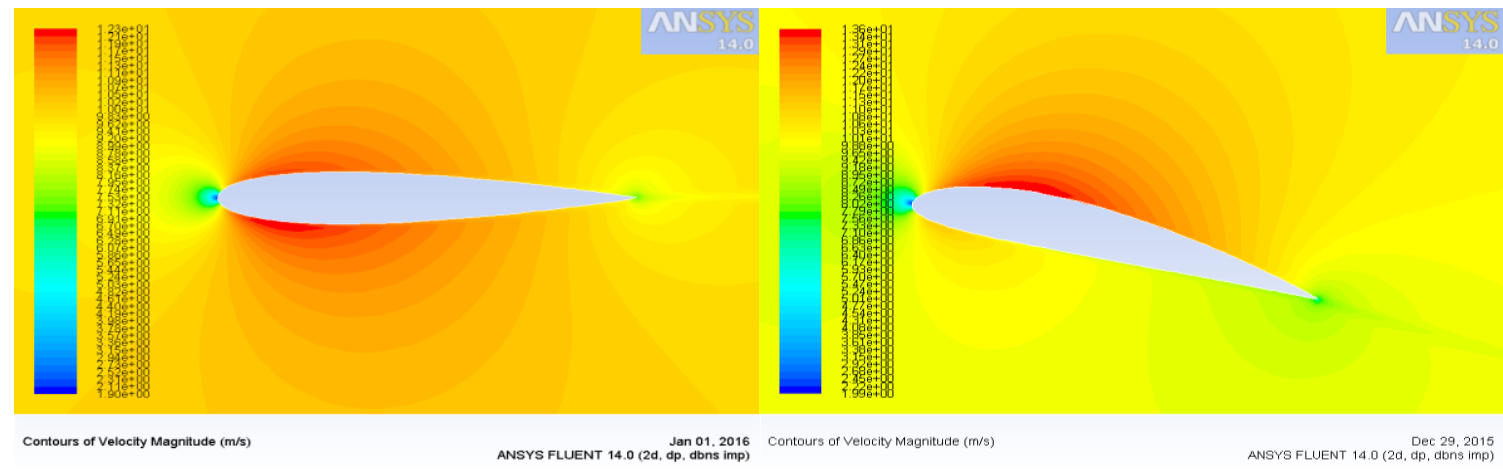

(a) $0^{\circ}$ angle of attack

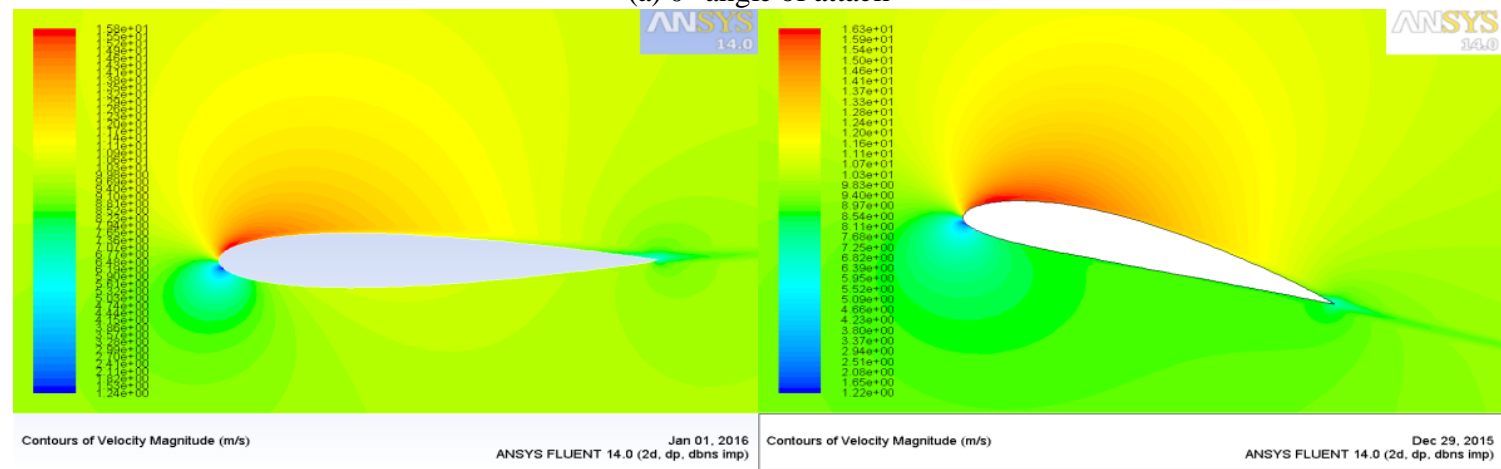

(b) $6^{\circ}$ angle of attack
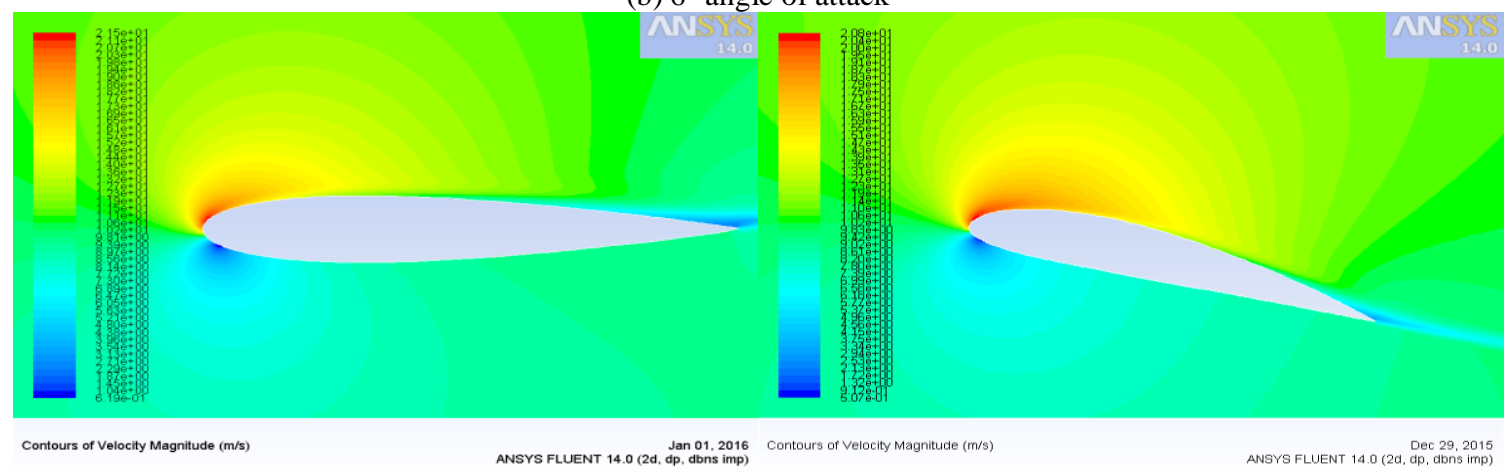

(c) $12^{\circ}$ angle of attack

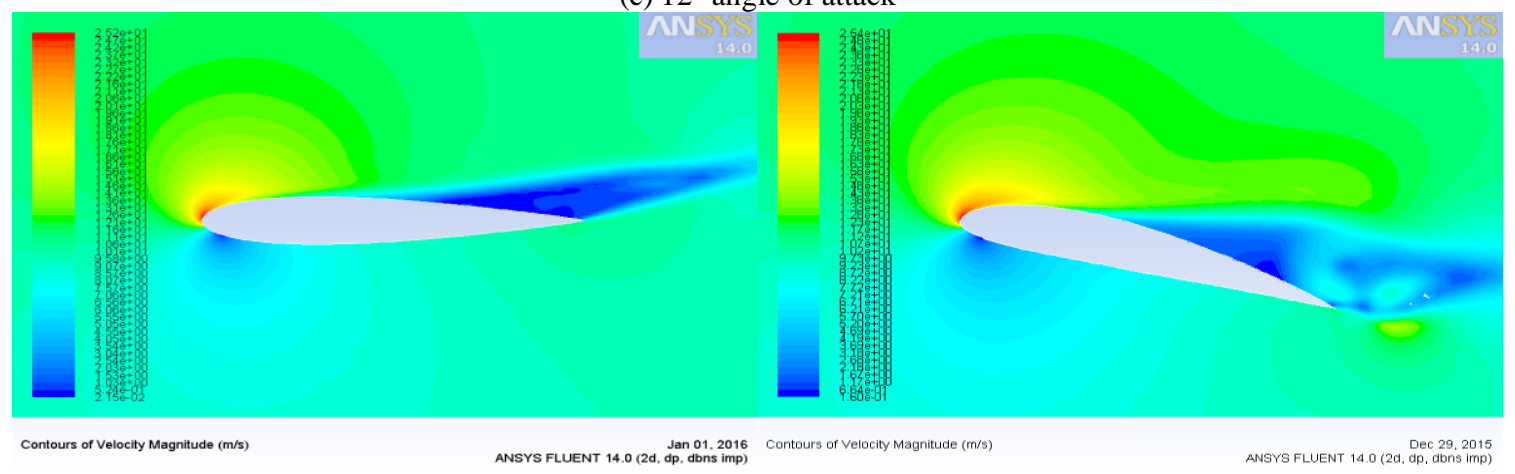

(d) $18^{\circ}$ angle of attack

Figure 5: Contours of velocity magnitude for NACA 0015 and NACA 4415. 


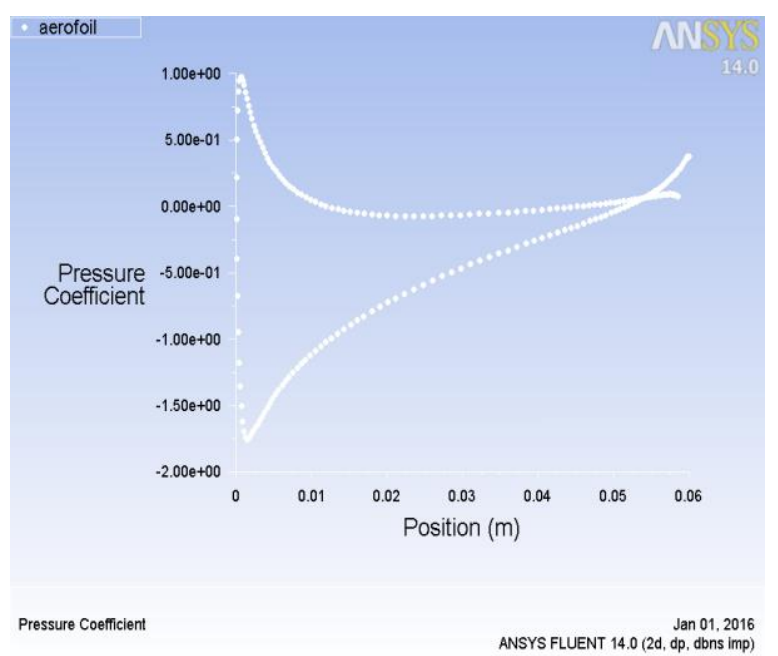

(a)

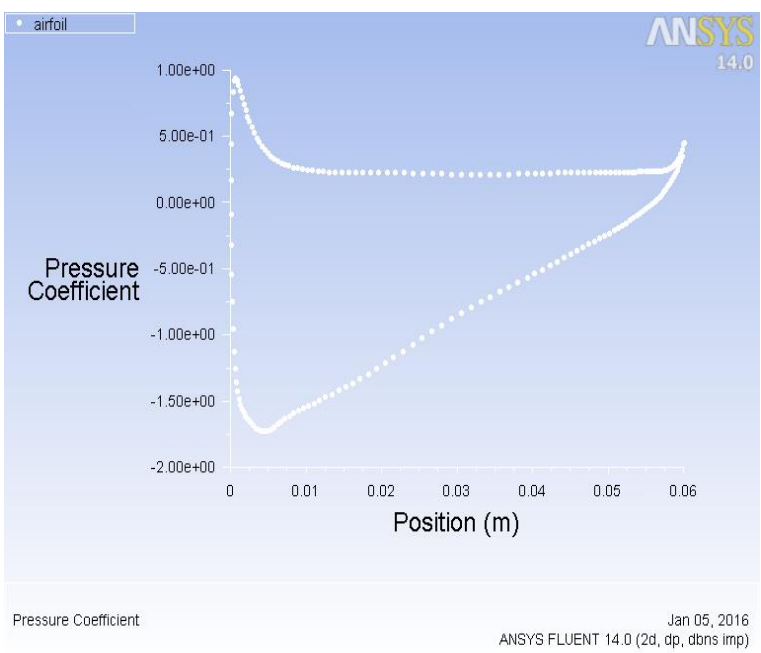

(b)

Figure 6: Pressure coefficient vs position of chord length curve for (a) NACA 0015; (b) NACA 4415.

For AOA greater than $12^{\circ}$ it will decreases slightly. Laminar flow is transition turbulence flow and pressure distribution changed around $16^{\circ}$ angle of attack so lift coefficient began to decrease. At $12^{\circ}$ AOA static pressure is more for NACA 4415 profile. At $18^{\circ}$ AOA significant turbulence and flow separation is visualized through the figure. It also has significant turbulence and flow separation at $18^{\circ} \mathrm{AOA}$ with presence of vortex of air appeared at the trailing section. The shape of the stream line indicates a change from a deficit to an excess condition across the vortex core at the leading edge.

\subsection{Contours of Velocity Magnitude}

Static pressure increases at the lower surface of the aerofoil with increasing angle of attack while reversely velocity magnitude increases at the upper surface as depicted by contours of velocity magnitude in Figure 5. At low velocity, lower surface generates more lift, which is commonly experienced at asymmetrical aerofoil (NACA 4415) than symmetrical aerofoil (NACA 0015). A laminar boundary layer arises to develop at the leading edge. Thickness of the layer grows in downstream direction (towards trailing edge). NACA 0015 trailing edge at $6^{\circ}$ AOA shows a small area of separated flow or initiation of flow separation that become dominant for increasing AOA. NACA 4415 shows similar phenomenon near to at $12^{\circ}$ AOA but change rapidly for $18^{\circ}$ AOA. Laminar boundary becomes unstable at some distance from the leading edge and is unable to suppress disturbances imposed on it by surface roughness or fluctuations in the free stream. Contours of velocity components at $0^{\circ} \leq \alpha \leq 18^{\circ}$ are also shown. For both of the profile stagnation point at trailing edge moves slightly forward at low AOA and jumped promptly towards leading edge at stall angle for NACA 4415 than NACA 0015.

\subsection{Pressure Coefficient vs Position of Chord Length Curve at $6^{\circ}$ Angle of Attack}

The two curves distinctly in Figure 6 show that negative pressure at the lower surface of the aerofoil is superior to pressure in upside surface. However, it is noticed that area of negative 
pressure for NACA 4415 is greater than NACA 0015 for its straight line. Similar curve can be obtained for any AOA.

\subsection{Comparison of the Experimental and Numerical Data with Using Characteristic Curve of NACA 0015 and NACA 4415}

A comparison of the effect and performance of aerofoil section on the minimum drag with practical construction surfaces is very difficult because the quality of the surface has more effect on the drag than the type of section. Probably the best comparison can be obtained from pairs of models constructed at the same time by the same manufacturers. Figure 7 is the comparable curve between numerical and experimental data for lift and drag forced. It is found very accurate value comparing numerical value and it is described for cambered aerofoil. In Figure 7(a) for NACA 0015 it is seen that lift coefficient increases with the increases of AOA up to a certain limit then it decreases.Drag coefficient also increases with the increases of AOA experimentally and numerically value of drag coefficient remains very closest. For cambered aerofoil (NACA 4415) angle of stall occur at more than $18^{\circ}$ and for NACA 0015 stall of angle has occurred at $12^{\circ}$ shown by an arrow. The higher the angle of stall means it would be gathered and maintains more surface area at the same time it can quick response when increases needs lift force by increases drag force. After calculating the lift coefficient between experimental and numerical for both NACA 0015 and NACA 4415 it is determined that the percentage of error for NACA 0015 is $4.8 \%$ and for NACA 4415 is $8.67 \%$. It is seen that for NACA 0015 the percentage of error becomes less than NACA 4415.

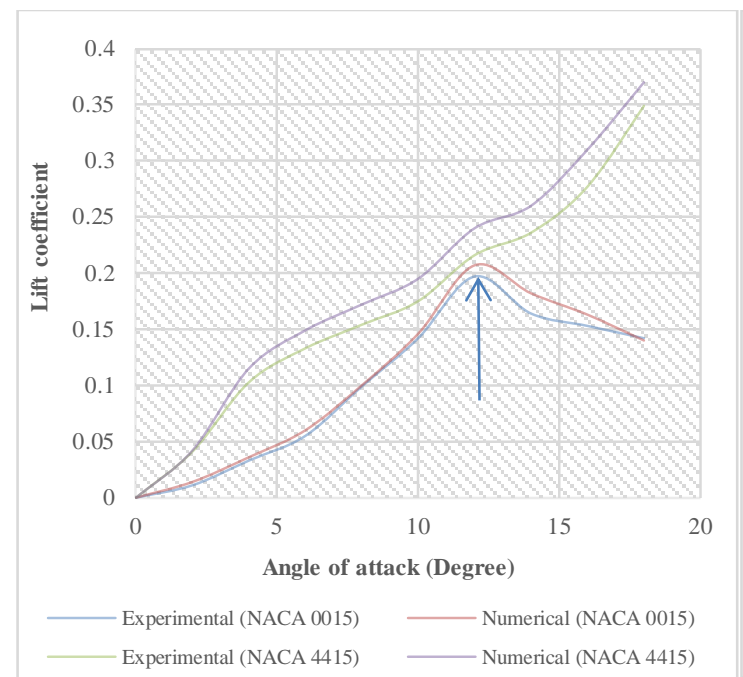

(a)

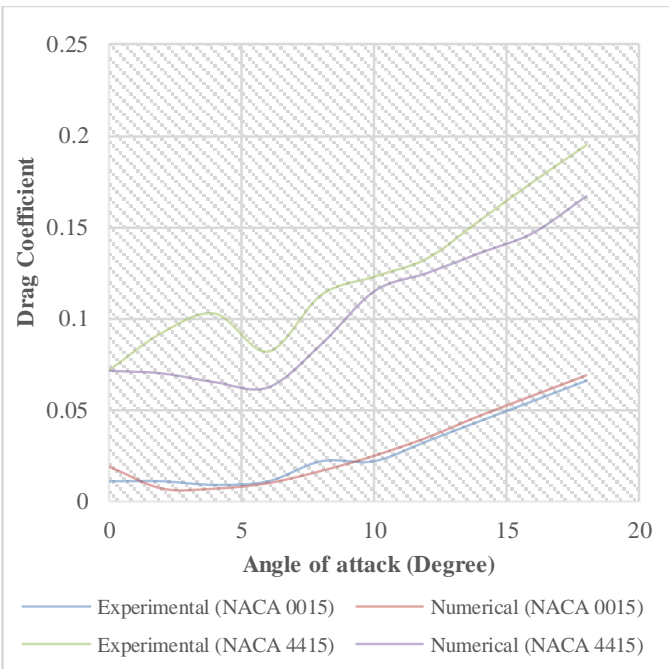

(b)

Figure 7: Variation of lift and drag coefficient w.r.to AOA for (a) NACA 0015; (b) NACA 4415.

\section{Conclusions and Recommendations}

A detailed experimental and numerical analysis of aerodynamic behavior for $0^{\circ} \leq \alpha \leq 18^{\circ}$ of NACA 0015 and NACA 4415 aerofoil profile is successfully tested in a subsonic wind tunnel assuming laminar flow governed by the Navier-Stokes incompressible flow equations. Outside the laminar boundary layer around aerofoil, effects of viscosity are assumed negligible and 
Bernoulli equation dominates instead of Navier-Stokes equation. With the intention of verify the accuracy and effectiveness, comparison is also made between two set of results. Experimental result and graph has slight mismatch with the obtaining graph from numerical research. From the above comparisons, it is clear that the cambered aerofoil NACA 4415 is distinctly the most efficient aerodynamic shape than symmetrical aerofoil NACA 0015. This works also helps to reduce the manual handling of aerofoil. At the same time, better result and comparison will give proper validation of the research. For same area of NACA 4415 can produce higher lift coefficient than NACA 0015. Stalling takes place for NACA 0015 at between $10^{\circ}<\alpha \leq 15^{\circ}$ AOA. The stalling phenomena responsible for introducing flow separation. A large negative pressure created on the NACA 4415 than NACA 0015, which accounts for most of the lift. Again, the above work suggests some recommendation for further research.

It is suggested to make the mesh size as small as possible so that smallest eddy fluctuations could have been caught by the computations. Turbulent behavior and friction may be considered.

The simulation and modeling consisted only for aerofoil model of NACA 0015 and NACA 4415 type, but it is suggested to model all the other series profile. So that easier and precise of lift and drag coefficient could have been applied keeping in mind that the computational grid must not become large or it would require large amount of computation power and computation time.

\section{References}

[1] Ravikumar T., Prakash, S.B., Aerodynamic analysis of supercritical NACA SC (2)-0714 airfoil using CFD. Int. J. Adv. Tech. Eng. Sci., 02 (2014), Issue 07, 285-293.

[2] Mccroskey, W. J., Kutler, P., Bridgeman, J. O., Status and prospects of computational fluid dynamics for unsteady transonic viscous flows. NASA_NTRS_Archive_19850003729.

[3] Siva, V., Analysis of ground effect on a symmetrical airfoil. Int. J. Eng. Res. App., 5 (2015) Issue 10, (Part - 2), 40-42.

[4] Zerihan, J., Zhang, X., Aerodynamics of a single element wing in ground effect. J. Aircr. 37 (2000), No. 6, 1058-1064. DOI: 10.2514/2.2711.

[5] Carr, L. W., Mcalister, K. W., Mccroskey, W. J., Analysis of the development of dynamic stall based on oscillating airfoil experiments, NASA TN D-8382, Ames Research Center and U.S. Army Air Mobility R\&D Laboratory Moffett Field, Calif. 94035.

[6] Katam, V., Simulation of low-Re flow over a modified NACA 4415 airfoil with oscillating camber, Master's Thesis, University of Kentucky, 2005, Lexington, Kentucky, U.S., Paper 339.

[7] Wen-Chao, Y., Hui, W., Jian-Ting, Y., Ji-Ming, Y., Characterization of the flow separation of a variable camber airfoil. Chin. Phys. Lett., 29 (2012), No. 4, 04470.

[8] Ragni, D., Ferreira, C.S., Correale, G., Experimental investigation of an optimized airfoil for vertical-axis wind turbines, Wind Energ., (2014). DOI: 10.1002/We.17805.

[9] Garg, P., Soni, N., Aerodynamic investigation of flow field over NACA 4415 airfoil. Int. J. Adv. Res. Sci. Eng. Tech., 03 (2016), Issue 2, 1506-1512.

[10] Boschetti, P.J., Cárdenas, E.M., Amerio, A., Aerodynamic optimization of an UAV Design. 5th Aviation Technology Integration and Operation, Arlington, Virginia, 2005. DOI: 10.2514/6.20057399

[11] Umapathi, M., Soni, N., Comparative analysis of airfoil NACA 2313 and NACA 7322 using computational fluid dynamics method. Int. J. Sci. Prog. Res. 12 (2015), No. 4, 193-198.

[12] Spentzos, A., Barakos, G., Badcock, K., Richards, B., Wernert, P., Schreck, S., Raffel, M., CFD investigation of $2 \mathrm{D}$ and $3 \mathrm{D}$ dynamic stall. 4th Decennial Specialist's Conference on 
Aeromechanics, San Fransisco, California, January 21-23, (2004). The American Helicopter Society Int., Inc.

[13] Munday D., Jacoby J., Active control of separation on a wing with oscillating camber. AIAA J. Aircraft, 39 (2002), No. 1.

[14] Gerontakos, P., Lee, T., PIV study of flow around unsteady airfoil with dynamic trailing-edge flap deflection. Exp. Fluids, 45 (2008), 955-972. DOI:10.1007/s00348-008-0514-4.

[15] Morshed, K.N., Experimental and numerical investigations on aerodynamic characteristics of savonius wind turbine with various overlap ratios. Master of Science in Applied Engineering, Georgia Southern University, Statesboro, Georgia 30458, (2010).

[16] Abbott, I.H., Doenhoff, A.E.V., Stivers, Jr. L.S., Summary of airfoil data. Report No. 824, Langley Memorial Aeronautical Laboratory Langley Field, Va., pp. 1.

[17] Zhang, Y., Igarashi, Y., Hu, H., Experimental investigations on the performance degradation of a low-Reynolds-number airfoil with distributed leading edge roughness. 49th AIAA Aerospace Sciences Meeting Including the New Horizons Forum and Aerospace Exposition, 4-7 January 2011, Orlando, Florida; AIAA: 1102, (2011).

[18] Yang, Z., Haan, F. L., Hui, H., An experimental investigation on the flow separation on a lowReynolds-number airfoil. 45th AIAA Aerospace Sciences Meeting and Exhibit, Jan 8 - 11, 2007, Reno, Nevada AIAA-0275, (2007).

[19] Llorca, I.B., CFD analysis and assessment of the stability and control of a supersonic business jet. Royal Institute of Technology (Kth), Stockholm, Sweden, (2015).

[20] Chacón Rebollo, T., Lewandowski, R., Mathematical and numerical foundations of turbulence models and applications. Springer, New York, (2014). DOI: 10.1007/978-1-4939-0455-6_2.

[21] John D. Anderson, Jr., Computational fluid dynamics, The basics with applications, 1st ed., TataMcgraw Hill: USA, (1995), 3-93.

[22] Weber, J., The calculation of the pressure distribution over the surface of two-dimensional and swept wings with symmetrical aerofoil sections. R. \& M. No. 2918, (16,124), A.R.C. Technical, (1956).

[23] Barnard, R.H., Philpott, D.R., Aircraft Flight: a description of the physical principles of aircraft flight. Longman Group Limited; 4th ed.; Essex, England, (2010).

[24] Dinesh K.G., Vignesh K.R., Ajay K.M., Srikanth J. V. R., Soorya P., Numerical investigation of vicious drag reduction using riblets. Adv. Aerosp. Sci. App., 03 (2013), No. 2, 75-82.

[25] Cousin, H. S., Torres, R. F., Zabihian, F., Panta, Y., Design and analysis of fluid flows through PIV and CFD modeling. Proceedings of the 2015 ASEE North Central Section Conference; American Society for Engineering Education: Washington, America, (2015).

\footnotetext{
*Corresponding author.

E-mail address: rubel@ baust.edu.bd
} 